\title{
Associations between indicators of screen time and adiposity indices in Portuguese children is
}

\author{
Emmanuel Stamatakis ${ }^{\mathrm{a}, \mathrm{b}, \star}$, Ngaire Coombs ${ }^{\mathrm{a}, \mathrm{b}}$, Russell Jago ${ }^{\mathrm{c}}$, Augusta Gama ${ }^{\mathrm{d}, \mathrm{i}}$, Isabel Mourão ${ }^{\mathrm{e}}$, \\ Helena Nogueira ${ }^{\text {f,i }}$, Vítor Rosado ${ }^{\text {g, i }}$, Cristina Padez h,i \\ ${ }^{a}$ Department of Epidemiology and Public Health, University College London, London, UK \\ b PARG (Physical Activity Research Group), Division of Population Health, University College London, London, UK \\ ${ }^{c}$ Centre for Exercise, Nutrition \& Health Sciences, School for Policy Studies, University of Bristol, UK \\ ${ }^{\mathrm{d}}$ Faculty of Sciences, University of Lisbon, Portugal \\ ${ }^{\mathrm{e}}$ University of Tras-os-Montes and Alto Douro, Research Center in Health and Human Development, Portugal \\ ${ }^{\mathrm{f}}$ Department of Geography, University of Coimbra, Portugal \\ Tropical Research Institute, Lisbon, Portugal \\ ${ }^{\text {h }}$ Department of Life Sciences, University of Coimbra, Portugal \\ ${ }^{\mathrm{i}}$ Research Centre for Anthropology and Health, University of Coimbra, Portugal
}

\section{a b s t r a c t}

Keywords:

Epidemiology

Adiposity

Sedentary lifestyle

Child

Behaviour

Life style
Objectives. To examine associations between three types of screen time (TV, electronic games (EG), and personal computer (PC)) and two proxies of adiposity (body mass index (BMI) and sum of skinfolds) in children. Design. The sample comprised 17,509 children aged 2-13 years who participated in the 2009/10 Portuguese Prevalence Study of Obesity in Childhood.

Methods. Complex samples generalised linear models, using school as a cluster variable were ran separately for each combination of ST predictor and adiposity-related outcome, adjusting for covariates including age, sex, physical activity, diet, and parental factors. Missing values in predictors and covariates were imputed.

Results. Watching TV for $>2 \mathrm{~h}$ /day compared to b $1 \mathrm{~h}$ /day was associated with higher age- and sex-specific BMI standard deviation score (coefficient: $0.06,95 \%$ CI: 0.01 to 0.12 , linear trend $\mathrm{p}=0.008$ ) and sum of skinfolds (logged and back transformed $0.04,0.02$ to $0.07, \mathrm{p}=\mathrm{b} 0.001$ ). We also found weak evidence for an inverse association between PC and BMI.

Conclusions. Associations between ST and adiposity differ by both type of ST and type of adiposity marker. Only TV viewing was consistently associated with adiposity. Studies using a single adiposity marker looking at total screen time or total sedentary behaviour time may miss or confound type-specific associations.
Introduction

Childhood is when physical activity (PA) and sedentary behaviour (SB) habits begin to form (Hills et al., 2007). Screen-related indicators of SB may be associated with adiposity in children and adolescents,

Abbreviations: BMI, Body mass index; CSGLMs, Complex samples generalised linear models; EG, Electronic games; MI, Multiple imputation; PA, Physical activity; PC, Personal computer; SB, Sedentary behaviour; SDS, Standard deviation score; ST, Screen time; TV, Television

is Financial disclosure: This study was supported by a grant from the Fundação para a Ciência e Tecnologia FCOMP-01-0124-FEDER-007483. This report is also research arising from Career Development Fellowships (Dr Stamatakis and Dr Jago) supported by the National Institute for Health Research. The views expressed in this publication are those of the authors and not necessarily those of the NHS, the National Institute for Health Research or the Department of Health.

* Corresponding author at: Department of Epidemiology and Public Health, University College London, 1-19 Torrington Place, London WC1E 6BT, UK.

E-mail address: e.stamatakis@ucl.ac.uk (E. Stamatakis). independent of physical activity (Crespo et al., 2001; Ekelund et al., 2006; Lioret et al., 2007).

Screen time (ST) (e.g. TV viewing, playing electronic games, and time spent using a computer), is one of the most prevalent forms of SB. Over $45 \%$ of boys and $47 \%$ of girls in England spend more than $2 \mathrm{~h}$ a day watching TV on weekdays (The Health and Social Care Information Centre, 2009). In Portugal the proportion of children watching more than $2 \mathrm{~h}$ of TV a day on weekdays is $28 \%$ of boys and $26 \%$ of girls, rising to $75 \%$ and $74 \%$ at weekends (Jago et al., 2012). TV viewing constitutes about half of the total SB (The Health and Social Care Information Centre, 2009). There are indications that TV viewing may have stronger associations with health outcomes than other types of SB (Fulton et al., 2009; Martinez-Gomez et al., 2009; Rey-López et al., 2008; Wake et al., 2003), but many studies focus either on TV viewing alone (Ekelund et al., 2006; Gortmaker et al., 1996), or on an aggregate measure of SB (Elgar et al., 2005; Mitchell et al., 2009) making it possible that type-specific associations between SB and adiposity are being obscured (Wake et al., 2003). 
Common adiposity proxy measures include body mass index (BMI), hip to waist ratio, fat free body mass, and skinfolds. Inconsistencies between studies in measurement of both SB and adiposity may be behind null associations reported in two relatively recent studies (Fulton et al., 2009; Mitchell et al., 2009). For this reason, it is important that SB studies use multiple indices of adiposity, when possible.

The aim of the present study was to examine the associations between different types of ST and markers of adiposity in a large population sample of Portuguese schoolchildren. To our knowledge this is the first study to examine associations between childhood (type-specific) ST and adiposity in Portugal.

Methods

The full unabridged methods section can be found in Appendix B

Sample

Data are from the Portuguese Prevalence Study of Obesity in Childhood which is a cross sectional study conducted between March 2009 and January 2010 in mainland schools in Portugal. Details on sampling and response rates can be found elsewhere (Jago et al., 2012). Briefly, schools were randomly selected from the Ministry of Education database. Sampling was based on a sex- and age-specific proportionate stratified random design with district as the primary sampling unit. A total of 17,509 2-13 year old children were recruited. Response rate was $57.4 \%$. Ethical approval was given by Direcção Geral de Inovação e Desenvolvimento. Parental informed consent was obtained prior to data collection.

Measurements

The screen-viewing behaviour of the children was assessed by proxy parental questionnaires. Specifically, parents were asked to report the average number of hours per day that the child spent watching TV, using a personal computer (PC) and playing electronic games (EG) on a weekday, on a Saturday and on a Sunday. Response options were none, up to $1 \mathrm{~h}, 1,2,3,4 \mathrm{~h}$ and $5 \mathrm{~h}$ or more. Anthropometric measurements (skinfold thickness, height, weight) were taken at school by a trained technician. The questionnaires also inquired about weekday and weekend active play (running, jumping, playing football and cycling), which was recorded using the same categories as TV viewing.

Other information collected in the questionnaire included contextual factors (consumption frequency of unhealthy foods including sodas, iced tea, cakes, chocolate, hamburgers and pizza; number of fruit portions eaten per day; parental weight and height; number of hours of sleep per night; birth weight and breastfeeding duration); parental socioeconomic indicators (parental education; parental employment status) and parental perception of crime in the neighbourhood.

Data handling

Body mass index $\left(\mathrm{BMI}=\mathrm{kg} / \mathrm{m}^{2}\right.$ ) was calculated for all children and converted to an age- and sex-specific standard deviation score (SDS) using the Cole formula as detailed in Vidmar et al. (2004). The sum of skinfold outcome variable was calculated by taking the sum of the mean of the two readings for each skinfold (triceps, subscapular and suprailiac). For TV, PC, EG and active play time, the original categories were recoded into a proxy continuous variable: none was coded as 0 , up to $1 \mathrm{~h}$ as $0.5,1 \mathrm{~h}$ as $1,2 \mathrm{~h}$ as 2 , and so on. $5 \mathrm{~h}$ or more was coded as 5 . These proxy continuous variables were then weighted such that weekdays accounted for $5 / 7$ ths, and Saturdays and Sundays each accounted for $1 / 7$ th of the variable.

For our analyses, the proxy continuous daily $\mathrm{TV}$ viewing time variable was re-categorised into three categories: b 1 h/day, 1-2 h/day, and $>2$ h/day. Due to lower per day volumes, different cut-offs were used for PC and EG times (b0.5 h/day, 0.5-1 h/day, >1 h/day). An unhealthy food consumption score was created with higher numbers indicating higher frequency of consumption of unhealthy foods (range from 6 to 36).

Multiple imputation

No predictor variable had more than $17.6 \%$ of values missing. However because listwise deletion of cases with missing values led to $>50 \%$ exclusion, we performed multiple imputation (MI) of missing values in predictor variables using the MI procedure in SPSS (version 18), with linear regression as the type of imputation model. Main results are presented using the pooled outcomes of five imputed datasets while key analyses using the original dataset with listwise deletion can also be found in Appendix A.

Analyses

The association between each ST variable and each of the two adiposity indicators was examined using generalised linear models and multiple linear regression to determine the trend $\mathrm{p}$ value. Before analysis, regression assumptions were checked. We used the complex samples generalised linear model (CSGLM) procedure in SPSS 18 to produce results with robust standard errors that take into account clustering of participants by school. To improve normality the sum of skinfolds was logged and outliers outside 4 standard deviations of the mean were removed (114 cases).

Models were adjusted: 1) for age and sex; 2) additionally for all contextual, socioeconomic and parental covariates (average hours of sleep per night, birth weight, duration of breastfeeding, unhealthy diet score, number of pieces of fruit per day, perception of crime in the local area, mother's education and BMI, and father's education and BMI); and 3) additionally for time spent in active play per week. CSGLM coefficients indicate mean differences (in values for each adiposity indicator) between the reference category and each of the other screen viewing categories. The lowest category (b1 h/day for TV and total ST, b $0.5 \mathrm{~h} /$ day for PC and EG) is the reference category for the mean difference in the outcome (and associated confidence interval for the difference) in all CSGLMs.

As we found little appreciable evidence of age or sex interactions, all analyses were age- and sex-adjusted, but not stratified. All of the above models were mutually adjusted for TV, EG and PC times in the 3rd model.

Results

Sample characteristics

A larger proportion of respondents were in the lowest TV, EG and PC categories than in the highest category (37\% compared to $26 \%$ for TV time, $58 \%$ compared to $4 \%$ for EG time, and $48 \%$ compared to $7 \%$ for PC time). Table 1 presents the sample characteristics by level of TV viewing (prior to MI and exclusion of outliers) with case-wise deletion of missing values. Participants who watched TV $>2 \mathrm{~h}$ per day were more likely to be male, to be older, to have parents with b9 years of education, and to have parents who were more likely to consider the local area unsafe due to crime than participants who watched $\mathrm{TV} \leq 2 \mathrm{~h}$ per day. They were also more likely to have a higher BMI and sum of skinfolds, eat unhealthy food more frequently, and eat fewer pieces of fruit per day. TV viewing was also directly associated with active play.

Appendix Table A.2 compares characteristics of participants with and without missing values. Participants with a missing value for one or more predictor variables on average spend more time watching TV, and on EG and PC use, have lower parental education, and spend more time on active play non-than participants with no missing values ( $\mathrm{p}$ b 0.001 for all observations). Participants without missing values had higher BMI and sum of skinfolds (difference in mean BMI 0.22, p b 0.001 difference in mean sum of skinfolds $0.69, \mathrm{p}=0.002$ ). These differences imply that missing values are not missing at random, supporting the use of MI rather than listwise deletion.

\section{TV, EG and PC times and adiposity}

Table 2 shows the results from the models with TV as the main exposure. Higher levels of TV viewing per day ( 1 to 2 and $>2$ h) compared to lower TV viewing levels (b1 h) were associated with both higher BMI SDS and sum of skinfolds in all models. EG time was not associated with BMI SDS or with sum of skinfolds in any model (Table 3). Higher levels of PC time ( 0.5 to 1 and $>1 \mathrm{~h})$ compared to lower PC time (b $0.5 \mathrm{~h})$ were weakly positively associated with lower BMISDS, but not with sum of skinfolds (Table 4). 
Table 1

Sample characteristics by level of TV viewing: prior to multiple imputation, with case wise deletion of missing values. ${ }^{a}$ All variables were significantly associated with TV viewing at a $95 \%$ level apart from breastfeeding duration and birth weight.

\begin{tabular}{|c|c|c|c|c|c|c|c|c|c|c|}
\hline \multirow{3}{*}{\multicolumn{2}{|c|}{ Categorical variable $^{\mathrm{b}}$}} & \multicolumn{8}{|c|}{ Average TV viewing time per day (h) } & \multirow[t]{3}{*}{$\mathrm{p}$} \\
\hline & & \multicolumn{3}{|l|}{ b 1} & \multicolumn{2}{|l|}{1 to 2} & \multicolumn{3}{|l|}{$>2$} & \\
\hline & & $\%$ & \multicolumn{2}{|l|}{$\mathrm{n}$} & $\%$ & $\mathrm{n}$ & \multicolumn{2}{|l|}{$\%$} & $\mathrm{n}$ & \\
\hline \multicolumn{2}{|l|}{ Sex ( $\%$ male $)$} & 47.9 & 5501 & & 50.7 & 5410 & \multicolumn{2}{|l|}{50.8} & 3841 & 0.004 \\
\hline \multicolumn{2}{|l|}{ Active play ( $\%>1 \mathrm{~h}$ per day) } & 54.4 & 5170 & & 57.4 & 5085 & 62.9 & & 3593 & b. .001 \\
\hline \multicolumn{2}{|c|}{ Father's education ( $\% 9$ years or more) } & 80.0 & 5237 & & 77.1 & 5102 & 69.0 & & 3533 & b. .001 \\
\hline \multicolumn{2}{|c|}{ Mother's education ( $\% 9$ years or more) } & 87.4 & 5401 & & 85.2 & 5284 & \multirow{2}{*}{$\begin{array}{l}77.6 \\
59.9\end{array}$} & & 3723 & b. .001 \\
\hline \multicolumn{2}{|c|}{ Area crime (\% strongly disagree unsafe) } & 66.2 & 5135 & & 64.9 & 4947 & & 59.9 & 3450 & b. .001 \\
\hline Continuous variables ${ }^{c}$ & Mean & (SD) & $\mathrm{n}$ & Mean & (SD) & $\mathrm{n}$ & Mean & (SD) & $\mathrm{n}$ & $\mathrm{p}$ \\
\hline Age & 6.64 & $(2.06)$ & 5501 & 7.14 & (1.99) & 5410 & 7.42 & $(2.00)$ & 3841 & b. .001 \\
\hline BMI $\left(\mathrm{kg} / \mathrm{m}^{2}\right)$ & 17.0 & $(2.38)$ & 5496 & 17.3 & $(2.66)$ & 5408 & 17.6 & $(2.86)$ & 3841 & b. .001 \\
\hline Sum of skinfolds (mm) & 23.7 & (12.0) & 4853 & 25.4 & (13.4) & 4781 & 26.7 & (14.4) & 3404 & b. .001 \\
\hline Mother BMI $\left(\mathrm{kg} / \mathrm{m}^{2}\right)$ & 23.6 & (3.73) & 5147 & 23.8 & $(3.80)$ & 5047 & 24.5 & $(4.18)$ & 3523 & b. .001 \\
\hline Father BMI $\left(\mathrm{kg} / \mathrm{m}^{2}\right)$ & 26.0 & (3.24) & 4810 & 26.1 & (3.30) & 4648 & 26.4 & (3.58) & 3205 & b. .001 \\
\hline Hours of sleep per night & 10.4 & $(0.62)$ & 5316 & 10.3 & $(0.61)$ & 5239 & 10.3 & (0.65) & 3711 & b. .001 \\
\hline Breastfeeding duration (months) & 4.89 & $(4.52)$ & 4697 & 4.74 & $(4.47)$ & 4633 & 5.02 & $(5.18)$ & 3169 & 0.033 \\
\hline Birth weight (g) & 3200 & $(533)$ & 5269 & 3219 & $(514)$ & 5201 & 3212 & $(525)$ & 3654 & 0.199 \\
\hline Unhealthy diet score & 15.2 & $(3.22)$ & 4826 & 15.9 & $(3.25)$ & 4675 & 16.9 & $(3.68)$ & 3289 & b. .001 \\
\hline Portions of fruit/day & 1.89 & $(0.95)$ & 5255 & 1.83 & $(0.94)$ & 5151 & 1.79 & $(0.96)$ & 3602 & b. .001 \\
\hline
\end{tabular}

Data are from 2009/10 Portuguese Prevalence Study of Obesity in Childhood.

${ }^{a}$ Respondents are included if they have valid data for the variable of interest (e.g. sex) and the stratification variable (TV viewing) regardless of missing data for any other variables. This results in a different number of respondents for each variable.

b Chi Square was used to test significance of association between categorical variables and TV viewing.

c ANOVA was used to test significance of association between continuous variables and TV viewing.

Comparison of imputed and non-imputed results

Appendix Table A.3 presents results from the non-imputed, listwise deleted data. There were no appreciable differences in the direction and magnitude of the observed associations with TV viewing as the main exposure. The weak inverse association of PC time seen in the imputed results with BMI was not present in the non-imputed analyses.

\section{Discussion}

This study uses unique data on a large population sample of schoolchildren living in Portugal to examine the associations between different indicators of screen time and two proxies of adiposity, BMI and sum of skinfolds. TV viewing showed consistent direct associations with both of these adiposity proxies, and BMISDS was inversely associated with PC time (models 2 and 3). Compared to children who watched $\mathrm{b} 1 \mathrm{~h}$ of TV per day, children who watched $\geq 2 \mathrm{~h}$ of TV had a sum of skinfolds $0.041 \mathrm{~mm}$ higher, and a BMI SDS 0.067 units higher (fully adjusted model). Although such effect sizes appear relatively small in terms of clinical importance, they have to be considered in the context of the large measurement error that all parental proxy physical activity and SB measures are bound to contain (Loprinzi and Cardinal, 2011). Non-differential inaccurate measurement of the exposures will most likely lead to attenuated associations with the outcomes (Ferrari et al., 2007; Spiegelman et al., 1997) and as such we can speculate that effect sizes would be much larger if we were to employ a more accurate measurement of ST, e.g. direct observation. Previous studies that take into account PA have also found associations between TV viewing and proxies of adiposity (Crespo et al., 2001; Ekelund et al., 2006; Lioret et al., 2007). Furthermore, studies have found non-TV types of SB to be unrelated to adiposity indicators, once PA and other factors are taken into account (Martinez-Gomez et al., 2009; Wake et al., 2003). Our results partially support this (sum of skinfolds did not show associations with non-TV ST; BMI SDS showed a borderline inverse associations with $\mathrm{PC}$ time) and indicate that associations between SB and adiposity vary by both the type of adiposity

Table 2

Multivariable-adjusted associations between TV viewing frequency and adiposity markers in Portuguese schoolchildren. At a $95 \%$ confidence level, significant linear trend were found between TV viewing and BMI SDS, and between TV viewing and sum of skinfolds in all models.

\begin{tabular}{|c|c|c|c|}
\hline TV viewing time per day & Model 1 coefficient $(95 \% \mathrm{CI})$ & Model 2 coefficient $(95 \% \mathrm{CI})$ & Model 3 coefficient $(95 \% \mathrm{CI})$ \\
\hline \multicolumn{4}{|l|}{ BMI SDS $(\mathrm{N}=17,474)$} \\
\hline 1-2 h compared to b $1 \mathrm{~h}$ & $0.074(0.031,0.117)$ & $0.054(0.014,0.095)$ & $0.057(0.016,0.098)$ \\
\hline$>2 \mathrm{~h}$ compared to b $1 \mathrm{~h}$ & $0.108(0.055,0.161)$ & $0.061(0.013,0.109)$ & $0.067(0.019,0.115)$ \\
\hline Trend $\mathrm{p}$ & b 0.000 & 0.014 & 0.007 \\
\hline \multicolumn{4}{|c|}{ Sum of skinfolds $(\mathrm{cm})(\mathrm{N}=15,358)$} \\
\hline $1-2 \mathrm{~h}$ compared to b $1 \mathrm{~h}$ & $0.031(0.016,0.046)$ & $0.025(0.011,0.04)$ & $0.027(0.012,0.041)$ \\
\hline$>2 \mathrm{~h}$ compared to $\mathrm{b} 1 \mathrm{~h}$ & $0.054(0.035,0.074)$ & $0.038(0.016,0.06)$ & $0.041(0.018,0.065)$ \\
\hline Trend $\mathrm{p}$ & b 0.000 & b 0.000 & b 0.000 \\
\hline
\end{tabular}

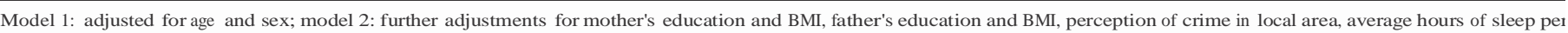

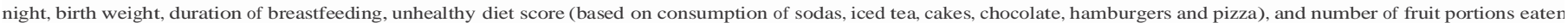
per week; model 3: further adjustments for time spent in active play per week.

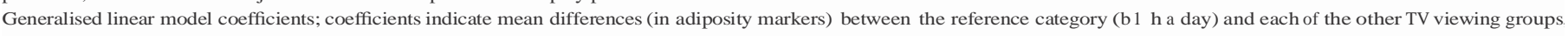
e.g. a value of 0.06 indicates that a specific category had a mean score that is 0.06 units higher than the referent group.

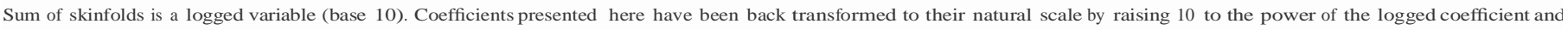
deducting 1.

Data are from 2009/10 Portuguese Prevalence Study of Obesity in Childhood. 
Table 3

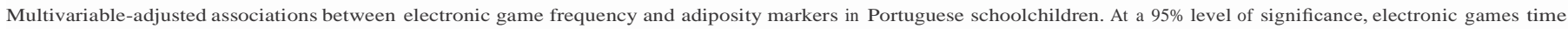
was not associated with BMI SDS or sum of skinfolds in any of the models.

\begin{tabular}{|c|c|c|c|}
\hline Electronic games time per day & Model 1 coefficient $(95 \%$ CI) & Model 2 coefficient $(95 \% \mathrm{CI})$ & Model 3 coefficient $(95 \% \mathrm{CI})$ \\
\hline \multicolumn{4}{|l|}{ BMI SDS $(\mathrm{N}=17,474)$} \\
\hline $0.5-1 \mathrm{~h}$ compared to b $0.5 \mathrm{~h}$ & $0.010(-0.033,0.053)$ & $0.004(-0.037,0.046)$ & $0.007(-0.034,0.049)$ \\
\hline$>1 \mathrm{~h}$ compared to b $0.5 \mathrm{~h}$ & $0.026(-0.069,0.122)$ & $-0.013(-0.097,0.070)$ & $-0.008(-0.091,0.075)$ \\
\hline Trend $\mathrm{p}$ & 0.421 & 0.877 & 0.983 \\
\hline \multicolumn{4}{|l|}{ Sum of skinfolds $(\mathrm{cm})(\mathrm{N}=15,358)$} \\
\hline $0.5-1 \mathrm{~h}$ compared to $\mathrm{b} 0.5 \mathrm{~h}$ & $-0.006(-0.022,0.01)$ & $-0.008(-0.024,0.009)$ & $-0.006(-0.023,0.011)$ \\
\hline$>1 \mathrm{~h}$ compared to $\mathrm{b} 0.5 \mathrm{~h}$ & $0.009(-0.025,0.044)$ & $-0.005(-0.034,0.025)$ & $-0.002(-0.031,0.028)$ \\
\hline Trend $\mathrm{p}$ & 0.915 & 0.436 & 0.592 \\
\hline
\end{tabular}

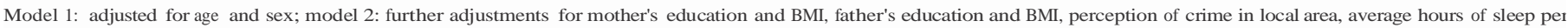

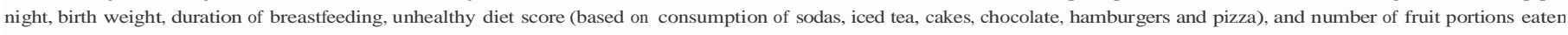
per week; model 3: further adjustments for time spent in active play per week.

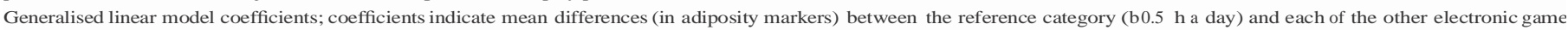
frequency groups, e.g. a value of 0.06 indicates that a specific category had a mean score that is 0.06 units higher than the referent group.

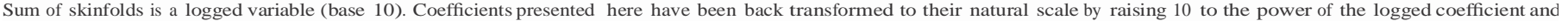
deducting 1 .

Data are from 2009/10 Portuguese Prevalence Study of Obesity in Childhood.

indicator and the type of SB. A study in Swedish adolescents found that adjustments for vigorous activity nullify the positive association between TV viewing and obesity (Ortega et al., 2007). As we were not able to classify our physical activity variable by intensity, we cannot verify or refute this finding.

It is unclear whether the associations between TV viewing and adiposity markers we observed are due to the sitting that TV viewing entails or due to other residual reasons. The general lack of an association between electronic games time and both adiposity indices and the weak inverse association between PC time and BMI that are also typically performed while sitting suggest that TV viewing might also reflect engagement in other behavioural risk factors, such as consuming high energy snack foods and/or the influence of TV advertisements on unhealthy behaviour (Cleland et al., 2008). In the present analyses we were only able to adjust for simple measurements of diet but not specifically for TV time snacking and hence residual dietary confounding might remain. Additionally, dietary underreporting (Lioret et al., 2007) might have compromised the ability of our dietary variables to fully capture the importance of diet. The study by Mitchell et al. (2009) that found that total (objectively-assessed, non-type specific) SB was not independently associated with obesity among a cohort of 5454 twelve-year old English children adds support to such an interpretation.

An alternative explanation of the differential associations we observed between types of ST and the adiposity proxies is differential measurement error of each screen time indicator and behaviour. That is, TV might be recalled and observed more accurately by the parents

than EG and PC use due to, for instance, viewing of specific programmes aiding recall and accuracy of time spent watching TV. Although we could locate no information specifically for parental proxy measures, TV viewing measurements have shown the highest validity among all SB measures in adults (Clark et al., 2009). Although screen time measures in children in general have acceptable test-retest reliability, their validity remains unknown (Lubans et al., 2011).

The strengths of this study are the large population sample and the availability of data on more than one screen-related behaviour and more than one adiposity indicator. To our knowledge, our study is the first to look at the associations between ST and skinfolds, a measure that is more closely correlated with actual body fat adiposity than BMI (Nooyens et al., 2007). A limitation of this study is lack of information on the characteristics of non-responders. We maximised statistical power by imputing missing data on covariables. Despite the small effect sizes we observed and within the study limitations, our results offer some support for childhood obesity interventions to target TV viewing, in addition to increasing physical activity. This is supported by previous research that has found interventions focusing on SB to be effective in reducing adiposity indicators in children, independently of physical activity (DeMattia et al., 2007; Epstein et al., 2000).

\section{Conclusion}

We found that TV viewing, but no other form of screen time, was positively associated with two common adiposity markers among

Table 4

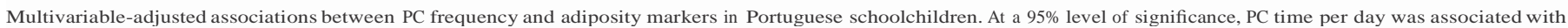
BMI SDS in models 2 and 3 only. PC time was not significantly associated with sum of skinfolds in any models.

\begin{tabular}{|c|c|c|c|}
\hline PC time per day & Model 1 coefficient $(95 \% \mathrm{CI})$ & Model 2 coefficient $(95 \% \mathrm{CI})$ & Model 3 coefficient $(95 \% \mathrm{CI})$ \\
\hline \multicolumn{4}{|l|}{ BMI SDS $(\mathrm{N}=17,474)$} \\
\hline $0.5-1 \mathrm{~h}$ compared to b $0.5 \mathrm{~h}$ & $-0.035(-0.077,0.006)$ & $-0.044(-0.084,-0.004)$ & $-0.043(-0.083,-0.003)$ \\
\hline$>1 \mathrm{~h}$ compared to b $0.5 \mathrm{~h}$ & $-0.028(-0.101,0.046)$ & $-0.054(-0.121,0.012)$ & $-0.05(-0.116,0.016)$ \\
\hline Trend $\mathrm{p}$ & 0.164 & 0.021 & 0.031 \\
\hline \multicolumn{4}{|c|}{ Sum of skinfolds $(\mathrm{cm})(\mathrm{N}=15,358)$} \\
\hline $0.5-1 \mathrm{~h}$ compared to b $0.5 \mathrm{~h}$ & $-0.006(-0.021,0.01)$ & $-0.006(-0.02,0.009)$ & $-0.005(-0.02,0.01)$ \\
\hline$>1 \mathrm{~h}$ compared to b $0.5 \mathrm{~h}$ & $0.003(-0.026,0.033)$ & $-0.004(-0.028,0.021)$ & $-0.002(-0.026,0.023)$ \\
\hline Trend $\mathrm{p}$ & 0.867 & 0.478 & 0.604 \\
\hline
\end{tabular}

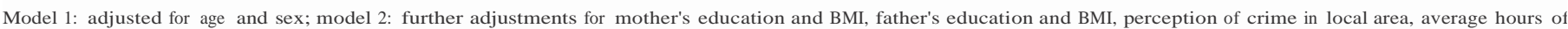

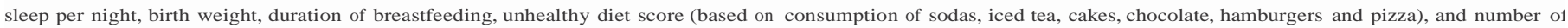
fruit portions eaten per week; model 3: further adjustments for time spent in active play per week.

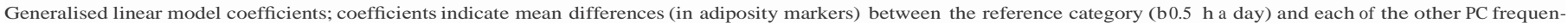
cy groups, e.g. a value of 0.06 indicates that a specific category had a mean score that is 0.06 units higher than the referent group.

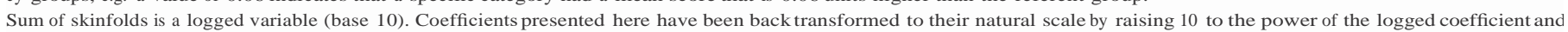
deducting 1 .

Data are from 2009/10 Portuguese Prevalence Study of Obesity in Childhood. 
Portuguese children. Future studies should investigate the role of TV viewing and other forms of sedentary behaviour in the development of obesity among youth using prospective designs with more than one marker of adiposity.

Conflict of interest statement

None of the authors have any conflict of interest.

\section{Acknowledgments}

This study was supported by a grant of the Fundação para a Ciência e Tecnologia FCOMP-01-0124-FEDER-007483. This report is also research arising from Career Development Fellowships (Dr Stamatakis and Dr Jago) supported by the National Institute for Health Research. The views expressed in this publication are those of the authors and not necessarily those of the NHS, the National Institute for Health Research or the Department of Health.

\section{References}

Clark, B.K., Sugiyama, T., Healy, G.N., Salmon, J., Dunstan, D.W., Owen, N., 2009. Validity and reliability of measures of television viewing time and other non-occupational sedentary behaviour of adults: a review. Obes. Rev. 10, 7-16.

Cleland, V.J., Schmidt, M.D., Dwyer, T., Venn, A.J., 2008. Television viewing and abdominal obesity in young adults: is the association mediated by food and beverage consumption during viewing time or reduced leisure-time physical activity? Am. J. Clin. Nutr. $87,1148-1155$.

Crespo, C.J., Smit, E., Troiano, R.P., Bartlett, S.J., Macera, C.A., Andersen, R.E., 2001. Television watching, energy intake, and obesity in US children. Arch. Pediatr. Adolesc. Med. 155, 360-365.

DeMattia, L., Lemont, L., Meurer, L., 2007. Do interventions to limit sedentary behaviours change behaviour and reduce childhood obesity? A critical review of the literature. Obes. Rev. 8, 69-81.

Ekelund, U., Brage, S., Froberg, K., et al., 2006. TV viewing and physical activity are independently associated with metabolic risk in children: the European Youth Heart Study. PLoS Med. 3, e488.

Elgar, F.J., Roberts, C., Moore, L., Tudor-Smith, C., 2005. Sedentary behaviour, physical activity and weight problems in adolescents in Wales. Public Health 119, 518-524.
Epstein, L.H., Paluch, R.A., Gordy, C.C., Dorn, J., 2000. Decreasing sedentary behaviors in treating pediatric obesity. Arch. Pediatr. Adolesc. Med. 154, 220-226.

Ferrari, P., Friedenreich, C., Matthews, C.E., 2007. The role of measurement error in estimating levels of physical activity. Am. J. Epidemiol. 166, 832-840.

Fulton, J.E., Dai, S., Steffen, L.M., Grunbaum, J.A., Shah, S.M., Labarthe, D.R., 2009. Physical activity, energy intake, sedentary behavior, and adiposity in youth. Am. J. Prev. Med. 37, S40-S49.

Gortmaker, S.L., Must, A., Sobol, A.M., Peterson, K., Colditz, G.A., Dietz, W.H., 1996. Television viewing as a cause of increasing obesity among children in the United States, 1986-1990. Arch. Pediatr. Adolesc. Med. 150, 356-362.

Hills, A.P., King, N.A., Armstrong, T.P., 2007. The contribution of physical activity and sedentary behaviours to the growth and development of children and adolescents. Sports Med. 37, 533-545.

Jago, R.J., Stamatakis, E., Gama, A., et al., 2012. Parent and child screen-viewing time and home media environment. Am. J. Prev. Med. 43, 150-158.

Lioret, S., Maire, B., Volatier, J.L., Charles, M.A., 2007. Child overweight in France and its relationship with physical activity, sedentary behaviour and socioeconomic status. Eur. J. Clin. Nutr. 61, 509-516.

Loprinzi, P.D., Cardinal, B.J., 2011. Measuring children's physical activity and sedentary behaviors. J. Exerc. Sci. Fitness 9, 15-23,

Lubans, D.R., Hesketh, K., Cliff, D.P., et al., 2011. A systematic review of the validity and reliability of sedentary behaviour measures used with children and adolescents. Obes. Rev. 12, 781-799.

Martinez-Gomez, D., Tucker, J., Heelan, K.A., Welk, G.J., Eisenmann, J.C., 2009. Associations between sedentary behavior and blood pressure in young children. Arch. Pediatr. Adolesc. Med. 163, 724-730.

Mitchell, J.A., Mattocks, C., Ness, A.R., et al., 2009. Sedentary behaviour and obesity in a large cohort of children. Obesity 17, 1596-1602.

Nooyens, A.C.J., Koppes, L.L.J., Visscher, T.L.S., et al., 2007. Adolescent skinfold thickness is a better predictor of high body fatness in adults than is body mass index: the Amsterdam Growth and Health Longitudinal Study. Am. J. Clin. Nutr. 85, 1533-1539.

Ortega, F.B., Ruiz, J.R., Sjöström, M., 2007. Physical activity, overweight and central adiposity in Swedish children and adolescents: the European Youth Heart Study. Int. J. Behav. Nutr. Phys. Act. 4, 61.

Rey-López, J.P., Vicente-Rodríguez, G., Biosca, M., Moreno, L.A., 2008. Sedentary behaviour and obesity development in children and adolescents. Nutr. Metab. Cardiovasc. Dis. 18, 242-251.

Spiegelman, D., Schneeweiss, S., McDermott, A., 1997. Measurement error correction for logistic regression models with an "alloyed gold standard". Am. J. Epidemiol. $145,184-196$.

The Health and Social Care Information Centre, 2009. Health Survey for England 2008: Volume 1 Physical Activity and Fitness.

Vidmar, S., Carlin, J., Hesketh, K., 2004. Standardizing anthropometric measures in children and adolescents with new functions for egen. Stata J. 4, 50-55.

Wake, M., Hesketh, K., Waters, E., 2003. Television, computer use and body mass index in Australian primary school children. J. Paediatr. Child Health 39, 130-134. 